\title{
A STUDY OF MESH OPTIMALITY CRITERIA IN ADAPTIVE FINITE ELEMENT ANALYSIS
}

\author{
E. OÑATE AND G. BUGEDA \\ International Centre for Numerical Methods in Engineering, ETS Ingenieros de Caminos, Canales y Puertos, \\ Universidad Politécnica de Cataluña, 08034 Barcelona, Spain
}

\begin{abstract}
The concepts of solution error and optimal mesh in adaptive finite element analysis are revisited. It is shown that the correct evaluation of the convergence rate of the error norms involved in the error measure and the optimal mesh criteria chosen are essential to avoid oscillations in the refinement process. Two mesh optimality criteria based on: (a) the equal distribution of global error, and (b) the specific error over the elements are studied and compared in detail through some examples of application.
\end{abstract}

KEY WORDS Adaptive mesh refinement Mesh optimality

\section{INTRODUCTION}

The evaluation of discretization error and the design of suitable meshes via adaptive mesh refinement (AMR) are nowadays two of the challenging issues in the finite element method (FEM).

The topic of error estimation and mesh adaptivity in the FEM is by no means new ${ }^{1-18}$. For a comprehensive review of the topic see the reference list of Chapter 14 of volume I of Reference 19.

In this paper a methodology for deriving AMR procedures is proposed. The basis of the approach is the decoupling of the concepts of error measure and mesh optimality criteria. This leads to the definitions of global and local error parameters from which the element refinement strategy can be simply obtained. Moreover, this allows to identify clearly the convergence rates of the global and local error norms, which strongly influence the expressions of the element refinement parameter. It is shown that an inaccurate evaluation of this important parameter can lead to oscillations in the refinement process.

The methodology proposed is particularized for two mesh optimality criteria using Zienkiewicz-Zhu error estimator ${ }^{1,3-6}$. First, the standard criterion of equal distribution of the global error over all the elements is studied. We will show here that a careful interpretation of the concepts of global error and optimal mesh leads in this case to an enhanced expression of the element refinement parameter slightly different from that typically used in the literature.

The second mesh optimality criterion studied is based in the equal distribution of the error per unit area or volume (i.e. the specific error). This strategy allows to concentrate more and smaller elements in zones where the gradients of the problem unknowns (i.e. stresses in structural problems) are higher, as it should be expected from the engineering point of view. 


\section{ERROR ESTIMATION AND ACCEPTABLE SOLUTION}

When dealing with adaptive mesh refinement finite element analysis the following two concepts should be clearly defined:

(a) Error estimator. Since the 'exact' solution is not known, a method to approximately evaluate the error of the finite element solution should be defined.

(b) Acceptable solution. A finite element solution is 'acceptable' if the estimated error satisfies some prescribed global and/or local conditions.

Both concepts are further extended in the next sections.

\section{Basic equations}

Let us consider the solution of a problem governed by a system of differential equations written in the general matrix form:

$$
\mathbf{A}(\mathbf{u})=0 \quad \text { in } \Omega
$$

\section{with boundary conditions:}

$$
\mathbf{B}(\mathrm{a})=\mathbf{0} \text { in } \Gamma
$$

where $\Omega$ is the area of the analysis domain (for $2 \mathrm{D}$ problems) and $\Gamma$ its contour. In (1) and (2) $u$ is a vector containing the problem unknowns, i.e. the displacements in a structural problem, the temperature in a thermal problem, the

We will define now the gradient vecto
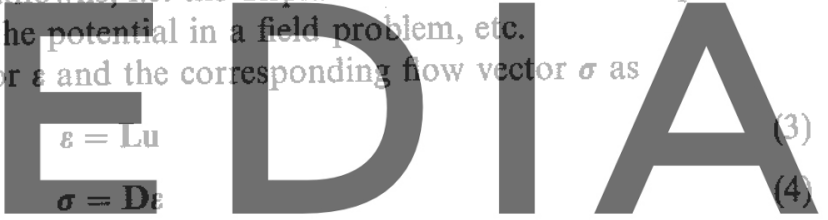

where $\mathbb{D}$ is a constitutive matrix depending on material properties.

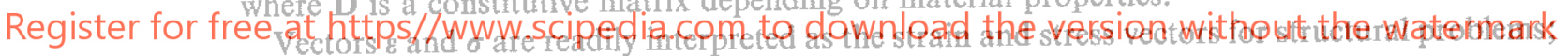

the temperature gradient and the heat flow vectors for thermal problems, etc.

For simplicity the fieids $\mathbf{u}, \boldsymbol{\varepsilon}$ and $\sigma$ will be identified hereafter with displacements, strains and stresses for a structural problem.

In the finite element solution the field $\mathbf{u}$ is interpolated in the standard form:

$$
\mathbf{u} \simeq \hat{\mathbf{u}}=\sum_{i=1}^{n} \mathbf{N}_{i} \overrightarrow{\mathbf{u}}_{i}=\mathbf{N} \tilde{\mathbf{u}}
$$

with

$$
\mathbf{N}_{i}=N_{i} \mathbf{I}
$$

where $N_{i}$ are shape functions, $\overline{\mathbf{u}}_{i}$ are the values of $\hat{\mathbf{u}}$ at the nodes of a finite element mesh and $n$ is the total number of nodes in the mesh ${ }^{19}$.

Substituting (5) in (1) and (2) and using standard weighted residual techniques or variational principles, leads (for static problems) to an equation system of the form:

$$
\mathbf{K} \overline{\mathbf{u}}=\mathbf{f}
$$

where the stiffness matrix $\mathbf{K}$ and the equivalent nodal force vector $\mathbf{f}$ are obtained by assembly of the element contributions ${ }^{19}$.

Once the nodal variables have been obtained from (6) the 'strains' and 'stresses' values are 
approximated as:

$$
\begin{gathered}
\varepsilon \simeq \hat{\varepsilon}=\sum_{i=1}^{n} \mathrm{LN}_{i} \overline{\mathrm{u}}_{i} \\
\sigma \simeq \hat{\sigma}=\sum_{i=1}^{n} \operatorname{DLN}_{i} \overline{\mathbf{u}}_{i}
\end{gathered}
$$

\section{Error estimator}

One of the most popular error estimators for elliptic problems is based on the error energy norm expressed as:

$$
\|\mathbf{e}\|=\left[\int_{\Omega}[\sigma-\hat{\sigma}]^{\mathrm{T}} \mathbf{D}^{-1}[\boldsymbol{\sigma}-\hat{\sigma}] \mathrm{d} \Omega\right]^{1 / 2}
$$

where $\sigma$ are the exact stresses, $\hat{\sigma}$ are the stress values obtained from the finite element solution (7b) and e is the displacements error.

Since the exact stresses are usually not known they are approximated by:

$$
\sigma \simeq \sigma^{*}=\mathbf{N}_{\sigma} \bar{\sigma}^{*}
$$

where $\mathbb{N}_{\sigma}$ are stress interpolating functions and $\bar{\sigma}^{*}$ are nodal stress values obtained by either simple nodal averaging of the finite element values, local globalleast square smoothing, or other adequate nodal stress with a lumped "mass"
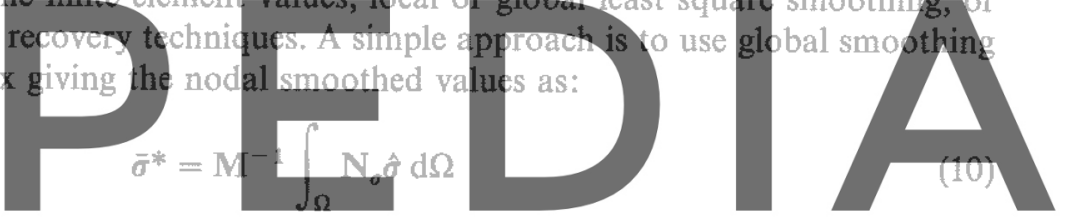

where $M$ is a diagonal matrix with $M_{i i}=\int_{\Omega} N_{\sigma_{3}} \mathrm{~d} \Omega$. Equation (10) can be obviously applied to

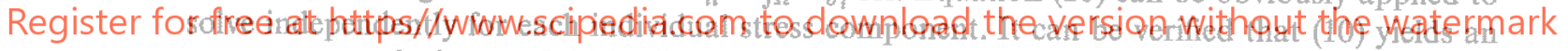
accurate smoothed stress field for linear elements and it is also adequate for quadratic elements providing some correction factors are introduced. A more accurate recovery technique has been recently proposed by Zienkiewicz and $\mathrm{Zhu}^{20}$ and this can be used as alternative.

The strain energy $U$ of the exact solution is estimated as:

$$
U=\|\mathbf{u}\|^{2} \simeq \int_{\Omega} \sigma^{* \mathrm{~T}} \mathbf{D}^{-1} \sigma^{*} \mathrm{~d} \Omega+\int_{\Omega}\left[\boldsymbol{\sigma}^{*}-\hat{\sigma}\right]^{\mathrm{T}} \mathbf{D}^{-1}\left[\boldsymbol{\sigma}^{*}-\hat{\sigma}\right] \mathrm{d} \Omega
$$

Both $\|\mathbf{e}\|$ and $\|\mathbf{u}\|$ norms can be evaluated as the sum of their respective element contributions as:

$$
\|\mathbf{e}\|^{2}=\sum_{i=1}^{n}\|\mathbf{e}\|_{i}^{2} \quad\|\mathbf{u}\|^{2}=\sum_{i=1}^{n}\|\mathbf{u}\|_{i}^{2}
$$

where $n$ is the total number of elements in the mesh.

\section{Definition of acceptable solution}

It is usually agreed that a solution is 'acceptable' if the two following conditions are satisfied:

(a) The global error in energy norm is not greater than a specified value of the total strain 
energy:

$$
\|\mathbf{e}\| \leqslant \eta\|\mathbf{u}\|
$$

where $\eta$ is the user's specified value of the permissible relative global error.

Equation (6) allows to define a global error parameter $\xi_{g}$ as:

$$
\xi_{g}=\frac{\|\mathbf{e}\|}{\eta\|\mathbf{u}\|}
$$

Clearly the values $\xi_{g} \leqslant 1$ denote satisfaction of the global error criterion, whereas $\xi_{g}>1$ indicates that further refinement is necessary.

A mesh refinement strategy based on $\xi_{g}$ only will lead to an uniform refinement $\left(\xi_{g}>1\right)$ or derefinement $\left(\xi_{g}<1\right)$ of all element sizes (with the usual corrections to preserve the dimensions of the analysis domain). A local error indicator is therefore needed and this is defined next.

(b) The distribution of elements in the mesh satisfies a 'mesh optimality criterion', this local condition can be expressed as:

$$
\|\mathrm{e}\|_{i}=\|\mathrm{e}\|_{r_{i}}
$$

where $\|\mathrm{e}\|_{i}$ is the actual error norm in each element $i$ and $\|\mathbf{e}\|_{r_{i}}$ is the 'required' error norm in the element. The local error parameter $\bar{\xi}_{i}$ is defined as:

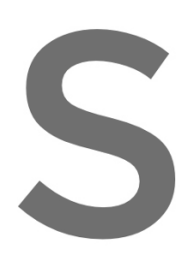

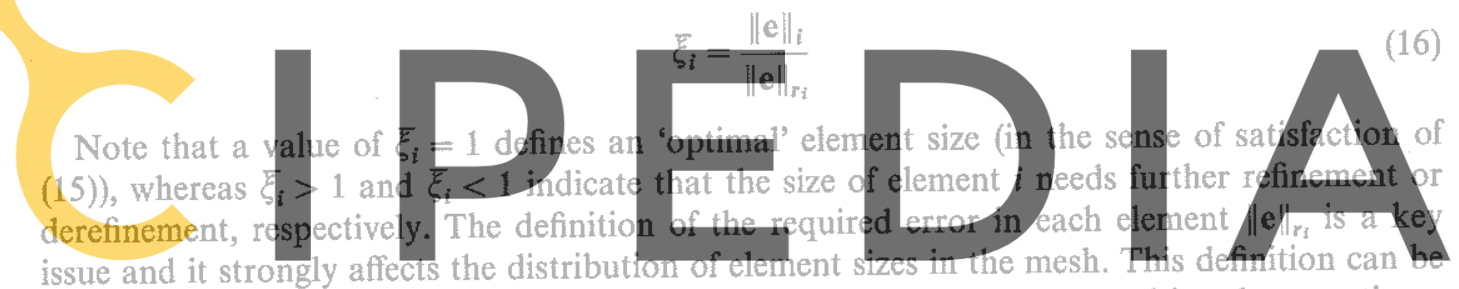
based on different mesh optimality criteria and some of these are presented in a later section.

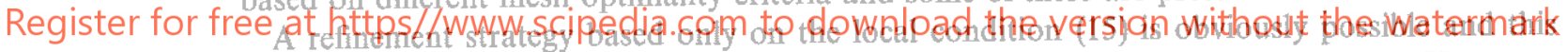
will imply the definition of appropriate bounds in the required element error norm. However, in practice it is usual to restrict the global error bound using condition (13). Nevertheless, other alternatives like a local limitation (i.e. $\|\mathbf{e}\|_{i} \leqslant \eta\|\mathbf{u}\|_{i}$ ) are possible and should be explored.

\section{Element refinement parameter}

Generally in practice we will aim to satisfy both local and global conditions (a) and (b) defined in the previous section. This allows to define an element refinement parameter using (14) and (16) as:

$$
\xi_{i}=\xi_{i} \xi_{g}=\frac{\|\mathbf{e}\|\|\mathbf{e}\|_{i}}{\eta\|\mathbf{u}\|\|\mathbf{e}\|_{r_{i}}}
$$

The element refinement parameter $\xi_{i}$ was first introduced by Zienkiewicz and $\mathrm{Zhu}^{1}$ and since then it has been used by many authors as the basis for defining the new element size in a general AMR strategy ${ }^{1-18}$. The expression of $\xi_{i}$ given in (17) can be interpreted as the result of trying to satisfy the global and local error conditions in a successive manner. Equation (17) provides all the terms involved in this combined process and they could play individually a very different role as explained in the next section. 


\section{MESH OPTIMALITY CRITERIA AND AMR PROCEDURES}

Mesh optimality criterion based on the equal distribution of the global error

A very popular criterion for elliptic problems is that a mesh is 'optimal' if the distribution of the energy norm is equal between all elements ${ }^{1,3-6,19}$. On the basis of this assumption the required error for each element can be defined as the ratio between the global error and the total number of elements in the mesh. Thus noting that only the square of the error norm is additive (see (12)) we have:

$$
\|\mathbf{e}\|_{r_{i}}=\frac{\|\mathbf{e}\|}{\sqrt{n}}
$$

Combining (16) and (18) yields the expression of the local error parameter as:

$$
\xi_{i}=\frac{\|\mathrm{e}\|_{i}}{\|\mathrm{e}\| n^{-1 / 2}}
$$

\section{The element refinement parameter is obtained, viz. (17) as:}

$$
\xi_{i}=\bar{\xi}_{i} \xi_{g}=\frac{\|\mathrm{e}\|_{i}}{\eta\|u\| n^{-1 / 2}}
$$
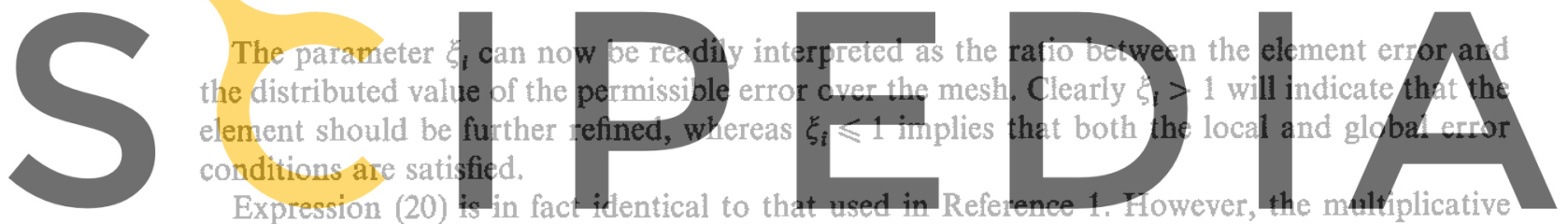

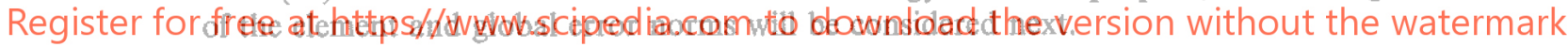

Let us assume that $m$ is the degree of the shape function polynomials in (5) ( $m=1$ for linear elements, $m=2$ for quadratic elements, etc.) and that only first derivatives of $u$ are involved in the strain operator $\mathbf{L}$ of (3). It is then easy to find for the global error norm (8):

$$
\|\mathbf{e}\|=\left[\int_{\Omega} O\left(h^{m}\right) \mathbf{D}^{-1} O\left(h^{m}\right) \mathrm{d} \Omega\right]^{1 / 2} \simeq O\left(h^{m}\right)
$$

where $h$ is the average element size of all the elements in the mesh. For the element error norm it is obtained:

$$
\|\mathbf{e}\|_{i}=\left[\int_{\Omega} O\left(h_{i}^{m}\right) \mathbf{D}^{-1} O\left(h_{i}^{m}\right) \mathrm{d} \Omega\right]^{1 / 2} \simeq O\left(h_{i}^{m}\right) \Omega_{i}^{1 / 2} \simeq O\left(h_{i}^{m+(d / 2)}\right)
$$

where $h_{i}$ and $d$ are, respectively, the existing element size and the number of dimensions of the problem $(d=1,2,3$ for $1 \mathrm{D}, 2 \mathrm{D}$ and 3D problems, respectively).

Equations (21) and (22) are essential to define the new element size $h_{i}$ in terms of the existing size using the expression:

$$
h_{i}=\frac{h_{i}}{\beta_{i}}
$$


with

$$
\beta_{i}=\xi_{g}^{1 / m} \xi_{i}^{2 /(2 m+d)}
$$

The expression of the element size parameter $\beta_{i}$ as given by (24) takes into account the different convergence rates of the element and global error norms.

Note that (24) can be interpreted as equivalent to applying a two step refinement (or derefinement) process in which the element sizes are first changed to fulfil the local mesh optimality criterion and then changed again to satisfy the global error criterion.

Zienkiewicz and $\mathrm{Zhu}^{1,3-6}$, followed by others ${ }^{7-10}$, use a simpler expression for $\beta_{i}$ based directly on the element refinement parameter $\xi_{i}$ as:

$$
\beta_{i}=\left(C \xi_{i}\right)^{1 / m^{\prime}}
$$

where $C$ is a relaxation factor (generally $C=1$ is taken ${ }^{4}$ ) and $m^{\prime}=m$ except for elements adjacent to singularities where $m=\lambda$ is used ( $\lambda$ being the singularity strength).

The authors have found that the computation of $\beta_{i}$ as given by (25) with $C=1$ and $m^{\prime}=m$ leads to a non-consistent mesh refinement. This is due to the violation of the convergence rate of the global and local error norms which enables the simultaneous satisfaction of both norms using a single element size parameter. This is shown by an oscillatory re- and de-refinement of some mesh zones in the AMR process.

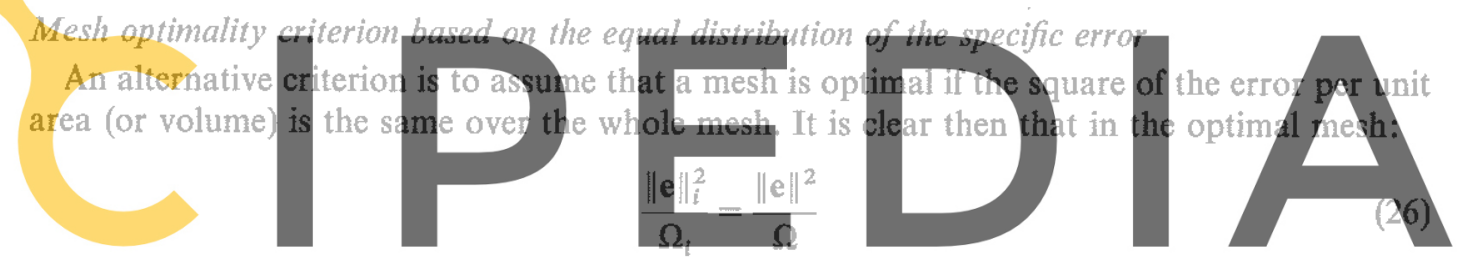

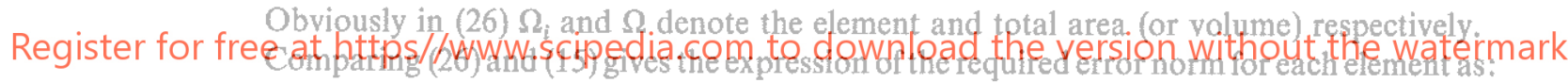

$$
\|\mathbf{e}\|_{r_{i}}=\|\mathbf{e}\|\left(\frac{\Omega_{i}}{\Omega}\right)^{1 / 2}
$$

The local error parameter $\xi_{i}$ is obtained now using (17) and (27) as:

$$
\bar{\xi}_{i}=\frac{\|\mathrm{e}\|_{i}}{\Omega_{i}^{1 / 2}}\left[\frac{\|\mathrm{e}\|}{\Omega^{1 / 2}}\right]^{-1}=\frac{\|\mathrm{e}\|_{i}}{\|\mathrm{e}\|}\left(\frac{\Omega}{\Omega_{i}}\right)^{1 / 2}
$$

The element refinement parameter $\xi_{i}$ is obtained from (14), (17) and (28) as:

$$
\xi_{i}=\bar{\xi}_{i} \xi_{g}=\frac{\|\mathbf{e}\|_{i}}{\eta\|\mathbf{u}\|}\left(\frac{\Omega}{\Omega_{i}}\right)^{1 / 2}
$$

Note that (20) and (29) coincide if $\Omega / \Omega_{i}=n$, i.e. all elements are equal in size. This is, however, not the case for unstructured meshes which results in quite different mesh distributions for the two optimality criteria considered, as shown in the examples.

The way the optimal element error is now defined eliminates its dependence with the element area, and the convergence rate of the specific element error can be deduced from (22) to be:

$$
\frac{\|\mathbf{e}\|_{i}}{\Omega_{i}^{1 / 2}} \simeq O\left(h_{i}^{m}\right)
$$


Note the coincidence of the convergence rates of the local and global error norms (21) and (30). The new element size is obtained from (23) with the element size parameter $\beta_{i}$ given now by:

$$
\beta_{i}=\left(\bar{\xi}_{i} \xi_{g}\right)^{1 / m}=\left(\xi_{i}\right)^{1 / m}
$$

The expression for the element refinement parameter coincides with that given by (25) for $C=1$ and $m^{\prime}=m$. Note, however, that (25) was introduced in the context of the mesh optimality criterion based on the equal distribution of the global error. The different forms presented here clarify the correct expressions of $\beta_{i}$ to be used for each mesh optimality criterion chosen.

To our knowledge the criterion of equal distribution of the specific error was first introduced in the form given here by Bugeda ${ }^{13}$ and it has been used in the context of optimum structural design problems by Bugeda and Oliver ${ }^{14,15}$ and for the AMR analysis of structures by the author's group ${ }^{11,12,16,17}$. This criterion can also be shown to be equivalent to that of equal error in stresses mentioned earlier ${ }^{1}$.

\section{EXAMPLES}

Four examples have been chosen to compare the different AMR strategies presented: (1) thick cylinder under internal pressure, (2) cylindrical shell roof under self weight, (3) perforated cylindrical shell under axial loading, (4) incompressible potential flow around an airfoil. In all the examples the following notation is used for the different AMR strategies.
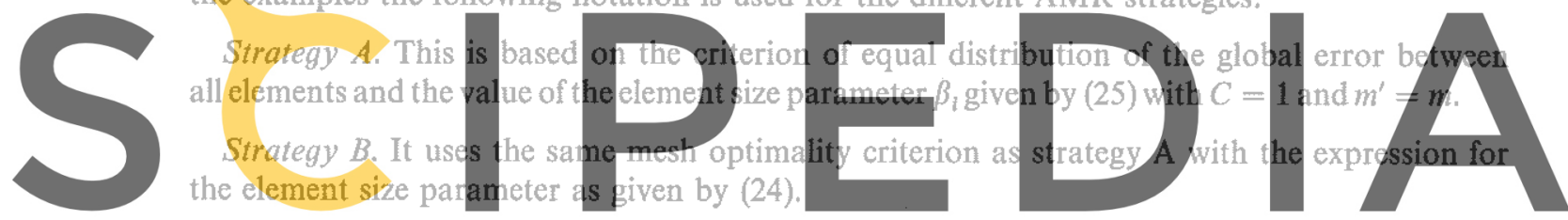

Strategy C. It is based on the criterion of equal distribution of the specific error with the

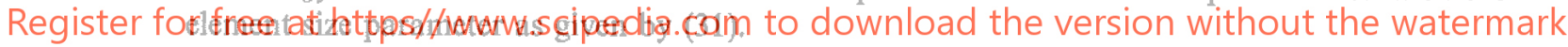

\section{Analysis of a thick circular cylinder under internal pressure}

The first example is the analysis of the thick circular cylinder under internal pressure shown in Figure 1. Due to the symmetry of the problem only a quadrant has been studied under plane strain conditions. Linear elastic behaviour has been assumed with $E=1.0 \times 10^{5}$ and $v=0.3$. A value of the permissible global error of $\eta=5 \%$ has been chosen. Standard 3 nodal linear triangular elements are used in the analysis. This example is typical of elliptic problems and it has many analogies in heat flow, ground water flow, etc. Figure 2 shows the sequence of refined meshes obtained with the three AMR strategies previously mentioned. First column of Figure 2 shows the results obtained with strategy A. Note the oscillations in the AMR process clearly shown by the alternative re- and de-refinement of some mesh zones.

Second column of Figure 2 shows the results obtained with strategy B. Note that the refinement oscillations disappear and the AMR process converges in a consistent manner.

Third column shows the results for strategy C. It can be clearly seen that: (a) the AMR process converges without oscillations, and (b) this AMR strategy concentrates more and smaller elements in the vicinity of the internal edge (where the error is greater due to the higher stress gradients), whereas in the rest of the mesh bigger elements than in the previous case are allowed. The price to be paid is the increase in the total number of elements with respect to strategies $A$ and $B$ for the same global accuracy as shown in Table 1. 


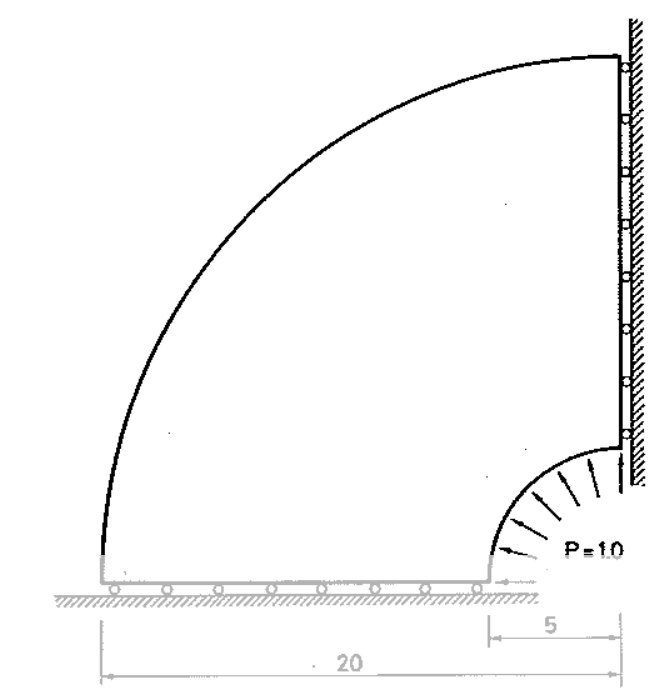

Figure 1 Thick circular cylinder under internal pressure-geometry and loads

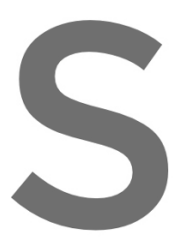

Table 1 show global error parameter deviation $\left(\xi_{1}^{2}\right)_{\sigma}$ over each this Table we deduce:

- all AMR strategies converge to the global permissible error chosen;

- the mean deviation of strategy A oscillates and does not converge to zero;

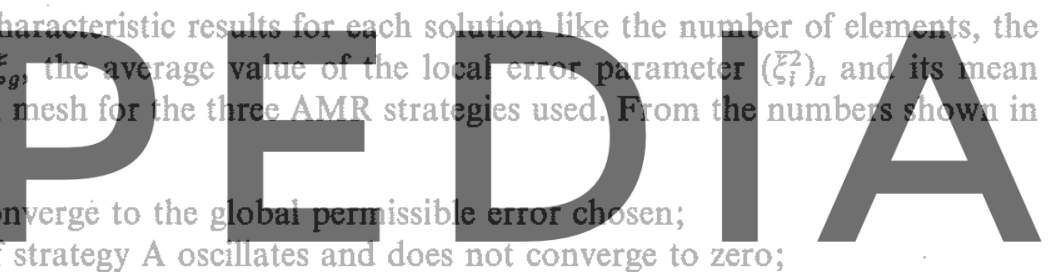

Register for free ptrategies B and C converge to an 'optimal mesh' characterized by the appropriate values

mesh is very different for these two AMR strategies.

Reference 16 shows results for the analysis of the same problem under diametrical loads with identical conclusions to the case presented here.

\section{Cylindrical shell roof under self weight}

The second example is the classical Scordelis cylindrical roof shown in Figure 3 together with the initial mesh of 48 triangular facet shell elements. The element used termed TLQL is based on an assumed shear strain formulation with linear interpolation for the displacements, quadratic interpolation for the rotations and linear interpolation for the shear strains ${ }^{11}$. The error energy norm in this case is expressed in terms of membrane forces $\mathbf{n}$, bending moments $\mathbf{m}$ and shear forces $\mathbf{s}$ as:

$$
\|\mathbf{e}\|^{2}=\int_{\Omega}\left\{[\hat{n}-\hat{\mathbf{n}}]^{\mathrm{T}} \mathbf{D}_{m}^{-1}[\mathbf{n}-\hat{\mathbf{n}}]+[\mathbf{m}-\hat{\mathbf{m}}]^{\mathrm{T}} \mathbf{D}_{b}^{-1}[\mathbf{m}-\hat{\mathbf{m}}]+[\mathbf{s}-\hat{\mathbf{s}}]^{\mathrm{T}} \mathbf{D}_{s}^{-1}[\mathbf{s}-\hat{\mathbf{s}}]\right\} \mathrm{d} \Omega
$$

where $\mathbf{D}_{m}, \mathbf{D}_{b}$ and $\mathbf{D}_{s}$ are the standard membrane, bending and shear constitutive matrices ${ }^{11}$.

The strain energy of the exact solution is estimated as:

$$
\|\mathbf{u}\|^{2} \simeq \int_{\Omega}\left\{\mathbf{n}^{* \mathrm{~T}} \mathbf{D}_{m}^{-1} \mathbf{n}^{*}+\mathbf{m}^{* \mathrm{~T}} \mathbf{D}_{b}^{-1} \mathbf{m}^{*}+\mathbf{S}^{* \mathrm{~T}} \mathbf{D}_{s}^{-1} \mathrm{~S}^{*}\right\} \mathrm{d} \Omega+\|\mathrm{e}\|^{2}
$$

where $(\cdot)^{*}$ denotes smoothed resultant stress values. 
Strategy A
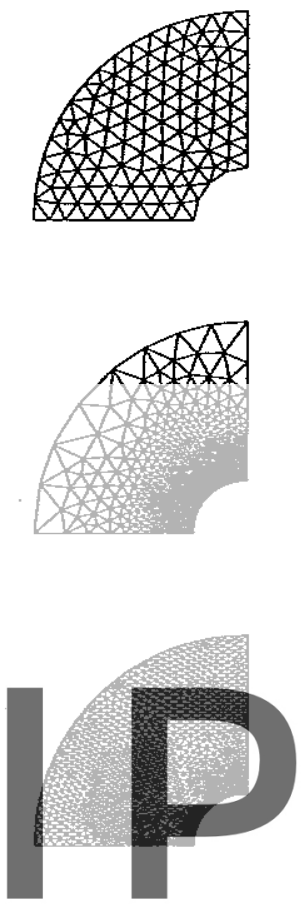

Strategy B
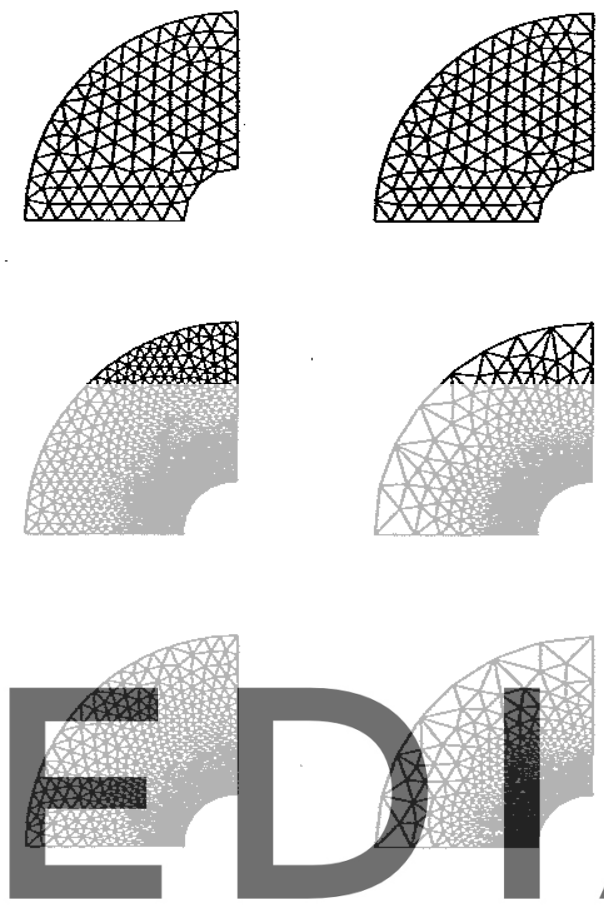

Register for free at https//www.scigediascom to downolde version withowthe watermark M3
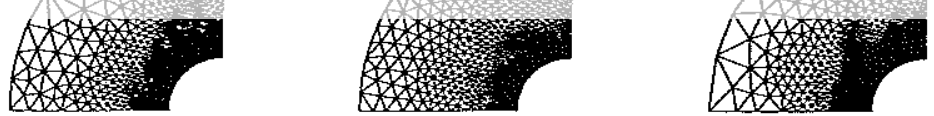

Figure 2 Thick circular cylinder under internal pressure-sequence of meshes obtained with AMR strategies $\mathrm{A}, \mathrm{B}$ and $\mathrm{C}$

Table 1 Thick circular cylinder under intemal pressure. Some statistical results of the AMR processes based on the equal distribution of the global error (strategies $A$ and $B$ ) and the specific error (strategy $C$ )

\begin{tabular}{|c|c|c|c|c|c|c|c|c|c|c|c|c|}
\hline & \multicolumn{4}{|c|}{ Strategy A } & \multicolumn{4}{|c|}{ Strategy B } & \multicolumn{4}{|c|}{ Strategy C } \\
\hline & NE & $\xi_{g}$ & $\left(\xi_{i}^{2}\right)_{a}$ & $\left(\xi_{i}^{2}\right)_{\sigma}$ & $\mathrm{NE}$ & $\xi_{g}$ & $\left(\xi_{1}^{2}\right)_{a}$ & $\left(\xi_{i}^{2}\right)_{0}$ & $\mathrm{NE}$ & $\xi_{g}$ & $\left(\bar{\xi}_{i}^{2}\right)_{\alpha}$ & $\left(\xi_{i}^{2}\right)_{\sigma}$ \\
\hline M0 & 200 & 4.133 & 1.000 & 8.401 & 200 & 4.133 & 1.000 & 8.401 & 200 & 4.133 & 1.190 & 15.37 \\
\hline M1 & 2820 & 0.919 & 1.000 & 8.469 & 2180 & 0.839 & 1.000 & 0.604 & 3028 & 0.884 & 2.224 & 2.071 \\
\hline M2 & 2711 & 0.907 & 1.000 & 2.197 & 1838 & 0.909 & 1.000 & 0.189 & 6359 & 0.796 & 1.204 & 0.163 \\
\hline M3 & 2758 & 0.839 & 1.000 & 4.484 & 1797 & 0.925 & 1.000 & 0.193 & 6026 & 0.835 & 1.206 & 0.151 \\
\hline
\end{tabular}




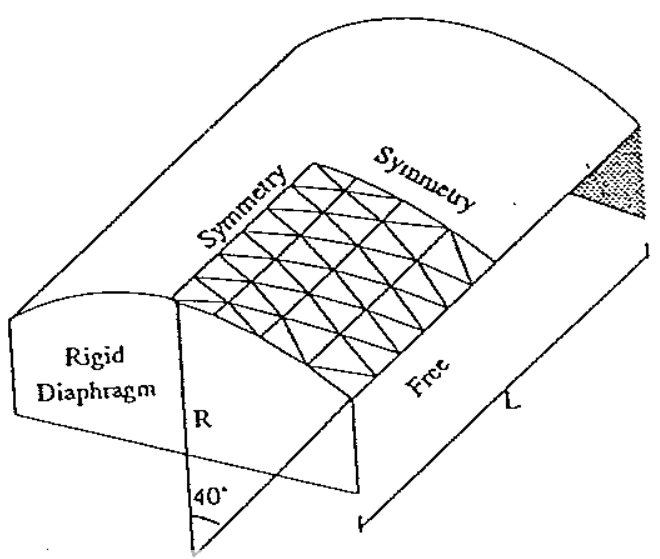

$$
\begin{aligned}
& L=600.0 \\
& R=300.0 \\
& \text { thickness }=3.0 \\
& E=3.0 \times 10^{6} \\
& \nu=0.0 \\
& \text { B.C. : in diaphragm } u=0, w=0 \\
& \text { Load : Self weight, } q=0.625 \\
& \text { Initial global error parameter } \xi_{g}=6.2295
\end{aligned}
$$

Figure 3 Cylindrical shell roof under self weight. Symmetric quadrant used in the analysis and initial mesh of 48 triangular assumed shear strain facet shell elements

\section{A value of the global permissible error $\eta=10 \%$ has been chosen in this case.}

Figure 4 shows the sequence of refined meshes obtained with the three AMR strategies used

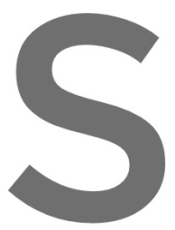

together with the

First column shows the results obtaine

process clearly shown by the alternative re-

Results labelec

for $\beta_{i}$ (strategy $\left.\mathbf{B}\right)$

in a consistent manner.

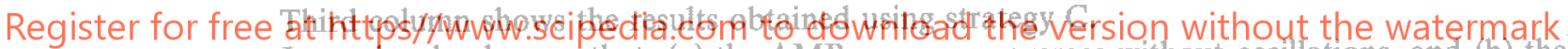

It can be clearly seen that: (a) the AMR process converges without oscillations, and (b) the

AMR strategy $\mathrm{C}$ concentrates more and smaller elements in the vicinity of the free edge (where

the error is greater due to the higher membrane stress gradients) whereas in the rest of the mesh bigger elements than in the previous case are allowed. The price to be paid is again the increase in the total number of elements with respect to strategies A and B for the same global accuracy.

Further details of this example and of the general AMR strategy for plates and shells can be found in References 11 and 12.

Analysis of a cylindrical shell with a circular hole under axial loading

Figure 5 shows the geometry, material properties of the shell and the initial mesh used. The analysis has been performed using the same facet shell element as in previous example. A value of the permissible global error $\eta=10 \%$ has been taken.

Figure 5 also shows the sequence of refined meshes obtained with the criterion of equal-distribution of the global error and the correct value for the element size parameter $\beta_{i}$ as given by (24) (strategy B), and also with the criterion of equal distribution of the specific error with $\beta_{i}$ given by (31) (strategy C).

Table 2 shows some characteristic results for the two AMR strategies used. From the numbers shown in this Table we deduce: both AMR strategies converge to the global permissible error chosen; both AMR processes converge to an 'optimal mesh', characterized by the appropriate values $\left(\xi_{i}^{2}\right)_{a}=1.0$ and $\left(\xi_{i}^{2}\right)_{\sigma}=0.0$. However, the number of elements and its distribution in each 
Strategy A

$N E=881 \xi_{g}=1.046$

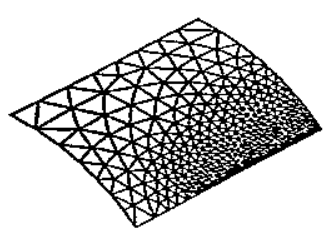

893

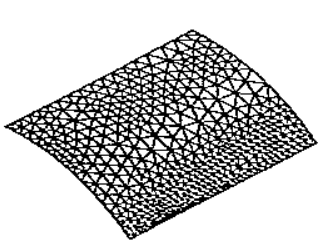

910
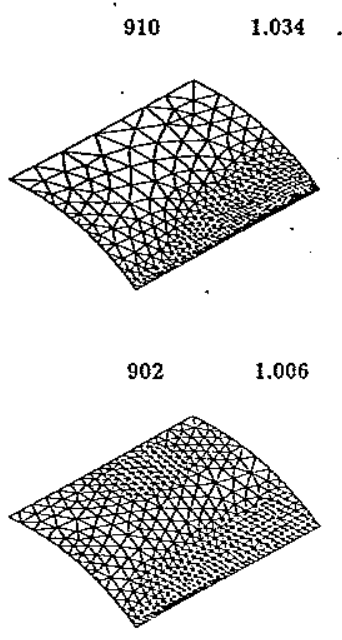

Strategy $B$

$N E=1.043 \xi_{g}=0.877$

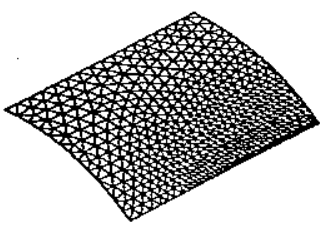

$749 \quad 1.007$

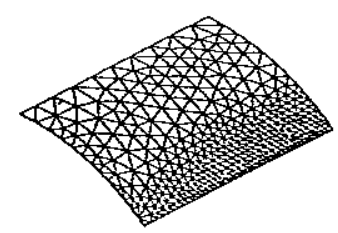

$736 \quad 1.006$

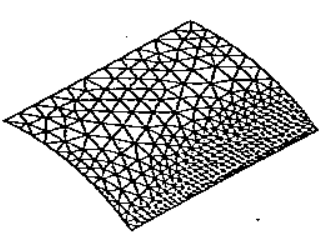

$747 \quad 1.005$

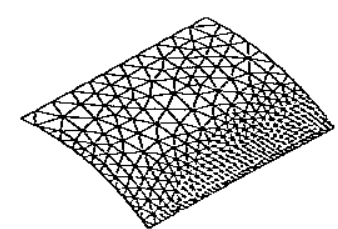

Strategy C

$N E=821 \quad \xi_{g}=1.113$

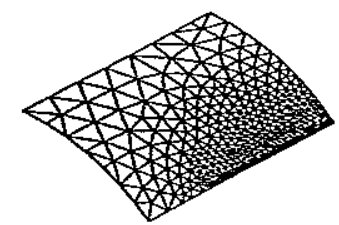

$1.594 \quad 1.183$

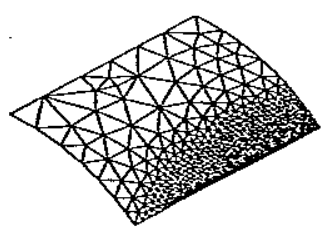

$1.878 \quad 0.991$

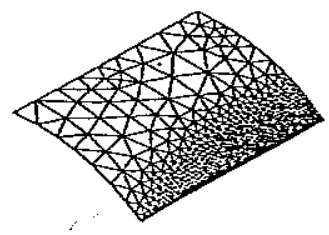

$2299 \quad 1.0002$

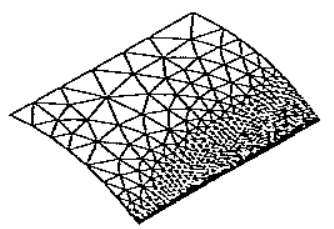

Figure 4 Symmetric quadrant of a cylindrical shell roof under self weight. Sequence of meshes obtained with AMR strategies A, B, and C. For each mesh is shown the number of elements (NE) and the value of the global error parameter $\xi_{g}$

Table 2 Symmetric quadrant of a tractioned cylindrical shell. Some statistical results of the AMR processes based on the equal distribution of the global error (strategy B) and the specific error (strategy $C$ )

\begin{tabular}{|c|c|c|c|c|c|c|c|c|}
\hline & \multicolumn{4}{|c|}{ Strategy B } & \multicolumn{4}{|c|}{ Strategy C } \\
\hline & $\mathrm{NE}$ & $\xi_{s}$ & $\left(\xi_{i}^{2}\right)_{\alpha}$ & $\left(\xi_{i}^{2}\right)_{\sigma}$ & $\mathrm{NE}$ & $\xi_{g}$ & $\left(\xi_{i}^{2}\right)_{a}$ & $\left(\xi_{i}^{2}\right)_{\sigma}$ \\
\hline Mo & 96 & 1.776 & 1.000 & 1.413 & 96 & 1.776 & 1.728 & 8.668 \\
\hline M1 & 234 & 1.078 & 1.000 & 0.613 & 393 & 0.993 & 1.545 & 3.432 \\
\hline M2 & 262 & 0.959 & 1.000 & 0.518 & 593 & 0.991 & 1.166 & 0.684 \\
\hline M3 & 266 & 0.968 & 1.000 & 0.384 & 729 & 0.979 & 1.050 & 0.381 \\
\hline
\end{tabular}




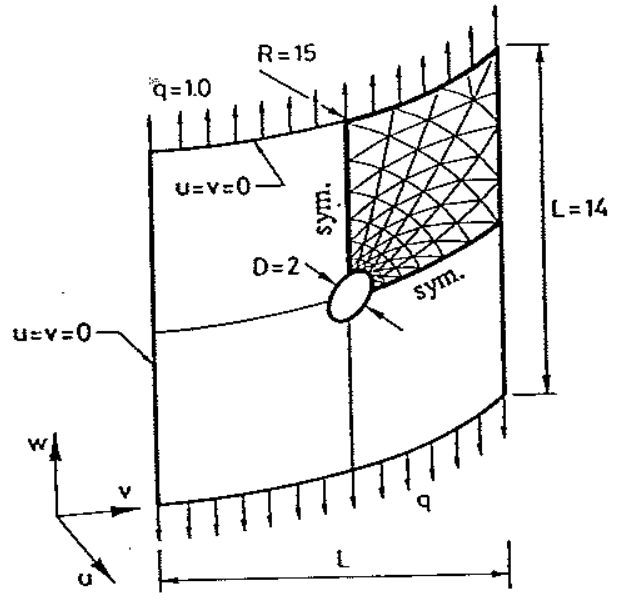

$$
\begin{aligned}
& E=2 \times 10^{6} \\
& \nu=0.3 \\
& \text { thickness }=0.086
\end{aligned}
$$

Initial mesh (M0) of

96 triangular elements

Strategy B
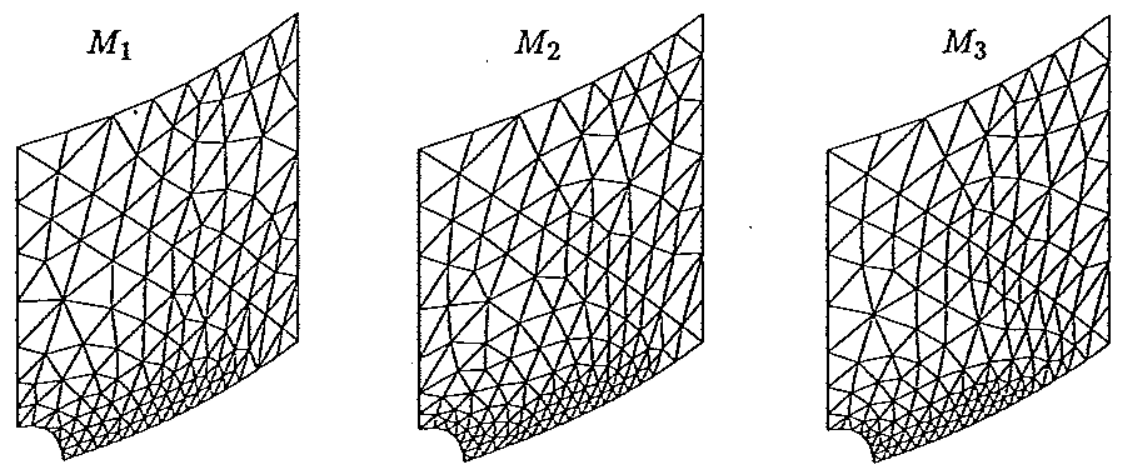

Strategy C
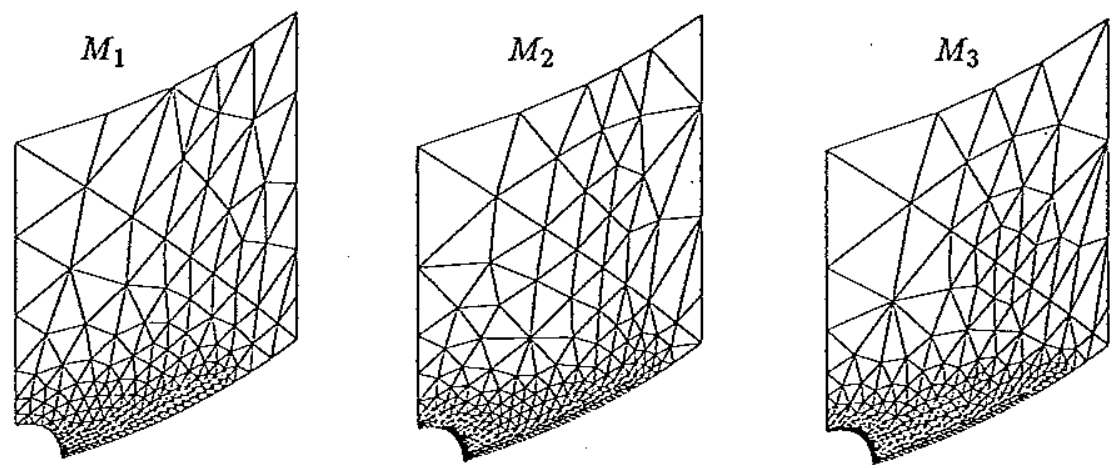

Figure 5 Analysis of a cylindrical shell under uniform traction. Sequence of meshes obtained with AMR strategies B and C 
mesh is very different for the two AMR strategies and again that based on the equal distribution of specific error (strategy C) tends to concentrate more and similar elements in the vicinity of the central hole where stress gradients are higher (see Figure 5).

\section{Potential flow around an airfoil}

The applicability of the AMR presented to fluid flow problems is shown in the analysis of the $2 \mathrm{D}$ flow around a classical Korn airfoil. An incompressible potential flow model with lifting has been used for this analysis. Details of this model can be found in Reference 17. The angle of attack has been taken equal to zero, the analysis domain has been discretized using standard 6 noded triangular elements. The exterior boundary of the domain has been defined at a distance of 10 chords from the profile.

The error energy norm and the energy of the exact solutions have been estimated as:

$$
\begin{gathered}
\|\mathbf{e}\|^{2} \simeq \int_{\Omega}\left[\mathbf{v}^{*}-\hat{\imath}\right]^{\mathrm{T}}\left[\mathbf{v}^{*}-\hat{v}\right] \mathrm{d} \Omega \\
\|\mathbf{u}\|^{2} \simeq \int_{\Omega} \mathbf{v}^{* \mathrm{~T}} \mathbf{v}^{*} \mathrm{~d} \Omega+\int_{\Omega}\left[\mathrm{v}^{*}-\hat{\vartheta}\right]^{\mathrm{T}}\left[\mathbf{v}^{*}-\hat{v}\right] \mathrm{d} \Omega
\end{gathered}
$$

In (34) $v$ is the velocity vector obtained from the final continuous potential solution as $\mathbf{v}=\nabla \phi$. Also, as usual, $\hat{v}$ and $\mathbf{v}^{*}$ denote velocities obtained directly from the finite element solution and after a nodal smoothing technique, respectively.

This case has been used to compare two different meshes with a similar number of degrees of freedom, but each one obtained using a different optimality criteria.

Figure 6 shows the mesh obtained after two remeshings using strategy $B$ and using a permissible global error of $\eta=0.02 \%$. This mesh has 2162 degrees of freedom and 1038 quadratic elements. In fact, the results from the first remeshing have a global error smaller than the permissible one but a new remeshing has been performed in order to get a better error distribution over the domain.

Figure 7 shows the mesh obtained after two remeshings using strategy $\mathrm{C}$ and using a permissible global error of $\eta=0.2 \%$. This mesh has 2133 degrees of freedom and 1019 quadratic elements. Again, the results from the first remeshing have a global error smaller than the permissible one.

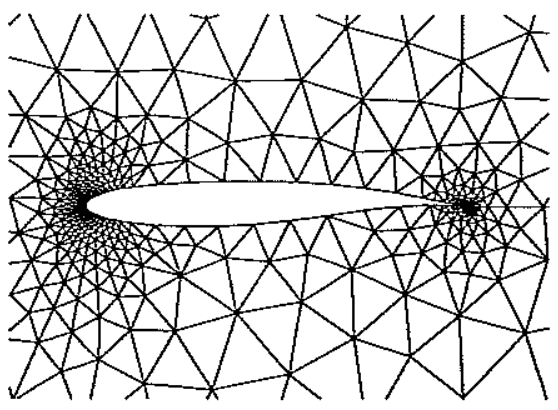

Figure 6 Analysis of a Korn airfoil. Mesh obtained with AMR strategy $\mathrm{B}(\eta=0.02 \%) .2162$ quadratic triangles and 1038 DOF

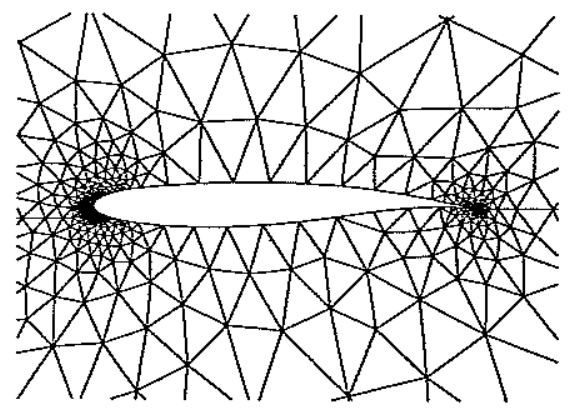

Figure 7 Analysis of a Korn airfoil. Mesh obtained with AMR strategy $\mathrm{C}(\eta=0.2 \%) .2133$ quadratic triangles and 1019 DOF 


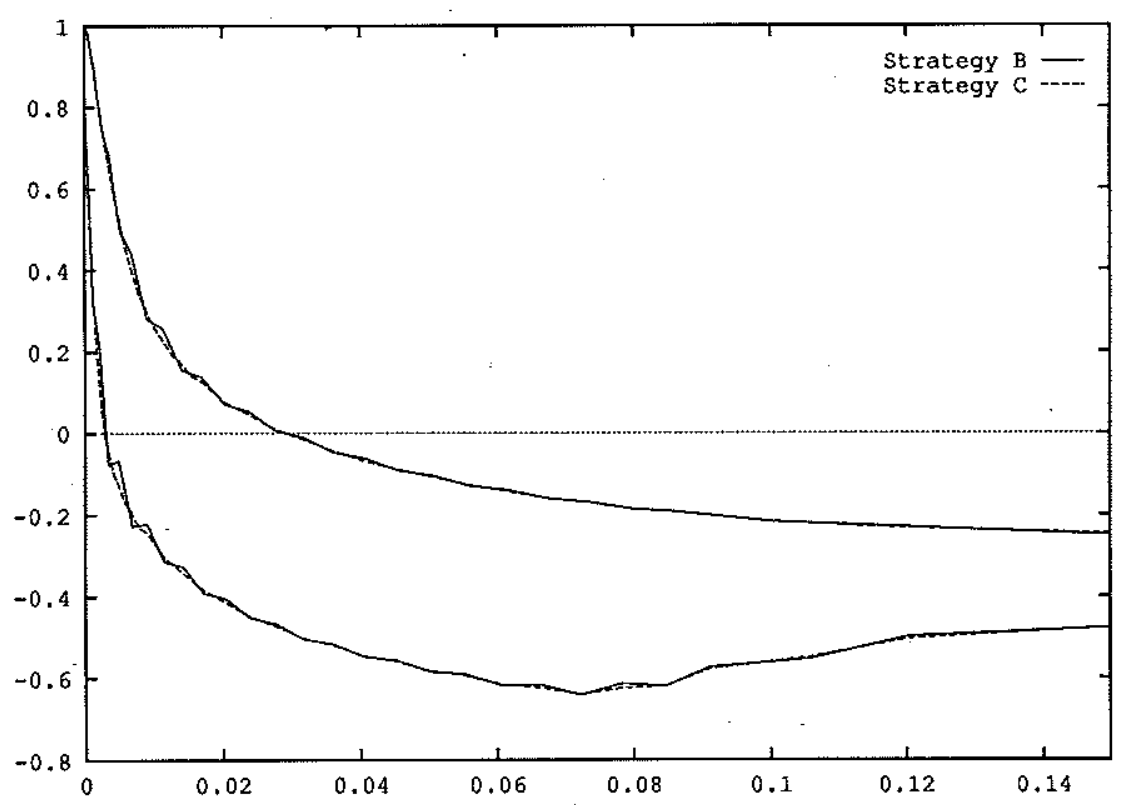

Figure 8 Analysis of a Korn airfoil. $C_{p}$ distribution around the leading edge obtained using strategies $\mathrm{B}$ and $\mathrm{C}$

The computational cost to solve the problem is very similar for both cases because the number of degrees of freedom for each one is practically identical. Looking at Figures 6 and 7 it can be observed that strategy $\mathrm{C}$ concentrates more and smaller elements in the vicinity of the leading edge (where the gradients of the velocities are higher), whereas in the rest of the domain the elements are bigger.

Figure 8 shows a plot of the $C_{p}$ distribution obtained with both meshes around the leading edge. The mesh obtained with strategy $B$ has a smaller global error norm (as prescribed), but this error is concentrated in the zone where the gradients of the velocities are higher, as it can be observed from the oscillations in Figure 8 . It is remarkable that although the mesh obtained with strategy $\mathrm{C}$ has a higher global error norm, the $C_{p}$ distribution around the leading edge is more accurate and no oscillations are observed.

From the last comments we conclude that for the same computational cost, strategy B leads to a smaller global error norm, but strategy $\mathrm{C}$ gives better results in the most interesting zones of the analysis domain.

\section{CONCLUDING REMARKS}

The definition of the mesh optimality criterion has proved to be an essential point in the design of an effective AMR process.

The use of the criterion of equal distribution of error over the elements leads to a 'smooth' distribution of the element sizes. This can be useful to prevent the use of meshes with large number of elements. However, this criterion is unable to concentrate elements in zones of high gradients only. It has been also shown that oscillations in the refinement process can appear in this case unless the element size parameter is consistently computed.

The criterion of equal distribution of specific error concentrates more and smaller elements in zones where important changes in the solution occur. This provides a more accurate solution 
in these regions which, in fact, are those of interest for the engineer. Therefore, this criterion seems to be the one to be recommended for practical purposes. The only drawback of this criterion is the large number of elements involved (this number tends to infinity if singularity points exist!). This can be overcome by appropriately prescribing the minimum element size or by introducing some limitations on the local error. These alternatives are currently explored by the authors.

\section{ACKNOWLEDGEMENTS}

The authors thank J. Castro and Prof. O. C. Zienkiewicz for many useful discussions and some of the ideas presented in this work.

\section{REFERENCES}

1 Zienkiewicz, O. C. and Zhu, J. Z. A simple error estimator and adaptive procedure for practical engineering analysis, Int. J. Num. Meth. Eng., 24, 337-357 (1987)

2 Zienkiewicz, O. C., Zhu, J. Z., Liu, 1. C., Morgan, K. and Peraire, J. Error estimates and adaptivity from elasticity to high speed compressible flow, in MAFELAP 87, (Ed. J. R. Shiteman), pp. 483-512, Academic Press, New York (1988)

3 Zhu, J. Z. and Zienkiewicz, O. C. Adaptive techniques in the finite element method, Commun. Appl. Num. Meth., 4, 197-204 (1988)

4 Zienkiewicz, O. C., Zhu, J. Z. and Gong, N. G. Effective and practical $h-p$ version adaptive analysis procedures for the finite element method, Int. J. Num. Meth. Eng., 28, 879-891 (1989)

5 Zienkiewicz, O. C. and Zhu, J. Z. The three R's of engineering analysis and error estimation and adaptivity, Comp. Meth. Appl. Mech. Eng., 82, 95-113 (1990)

6 Zienkiewicz, O. C. and Zhu, J. Z. Error estimates and adaptive refinement for plate bending problems, Int. J. Num. Meth. Eng., 28, 2839-2853 (1989)

7 Atamaz-Sibai, W. and Hinton, E. Adaptive mesh refinement with the Morley plate element, Proc. of NUMETA 90 Conf. Swansea, Vol. 2, pp. 1044-1057, Elsevier Appl Sci, London (1990)

8 Atamaz-Sibai, W., Hinton, E. and Selman, A. Adaptive mesh refinement with Mindlin-Reissner elements, Proc. 2nd Int. Conf. Computer Aided Analysis and Design of Concrete Structures, Zell-am-see, Austria, Vol. 1, pp. 303-315, Pineridge Press, Swansea (1990)

9 Selman, A., Hinton, E. and Atamaz-Sibai, W. Edge effects in Mindlin-Reissner plates using adaptive mesh refinement, Eng. Comput., 7, 217-227 (1990)

10 Hinton, E., Ozakşa, M. and Rao, N. V. R. Adaptive analysis of thin shells using facet elements, Int. Report $C R / 950 / 90$, Univ. College of Swansea (1990)

11 Onate, E., Castro, J. and Kreiner, R. Error estimations and mesh adaptivity techniques for plate and shell problems, 3rd Int. Conf. Quality Assurance and Standards in Finite Element Methods, Stratford-upon-Avon, NAFEMS (1991)

12 Oñate, E. and Castro, J. Adaptive mesh refinement techniques for structural problems, The Finite Element Method in the $90 \mathrm{~s}$ (E. Onate, J. Periaux and J. Samuelsson, eds.), Springer-Verlag \& CIMNE, Barcelona (1991)

13 Bugeda, G. Utilización de técnicas de estimación de error y generación automática de mallas en procesos de optimización estructural, $P h D$ Thesis, Universitat Polytècnica de Catalunya (1990)

14 Bugeda, G. and Oliver, J. Automatic adaptive remeshing for structural shape optimization, Eur. Conf. New Advances in Comput. Struct. Mech., Giens (1991)

15 Bugeda, G. and Oliver, J. A general methodology for structural shape optimization problems using automatic adaptive remeshing, $I n t . J . N u m$. Meth. Eng. (in press)

16 Bugeda, G. and Oñate, E. New adaptive techniques for structural problems, First Eur. Conf. Numerical Methods in Engineering, Brussels, Elsevier, Amsterdam (1992)

17 Bugeda, G. and Oñate, E. Adaptive mesh refinement techniques for aerodynamic problems, in Numerical Methods in Engineering and Applied Sciences (Eds. H. Alder, J. C. Heinrich, S. Lavanchy, E. Oñate and B. Suárez), CIMNE-Barcelona (1992)

18 Wu, J., Zhu, J. Z., Smelter, J. and Zienkiewicz, O. C. Error estimation and adaptivity in Navier-Stokes incompressible flow, Comput. Mech., 6, 259-271 (1990)

19 Zienkiewicz, O. C. and Taylor, R. L. The Finite Element Method, McGraw-Hill, New York, Vol. I (1989), Vol. II (1991)

20 Zienkiewicz, O.C. and Zhu, J. Z. Superconvergence derivative recovery techniques and a posteriori error estimation in the finite element method. Part I and Part II, Int. J. Num. Meth. Eng., 33, 1331-1382 (1992) 
